\title{
The dual of the James tree space is asymptotically uniformly convex
}

\author{
by \\ Maria Girardi (Columbia, SC)
}

Abstract. The dual of the James tree space is asymptotically uniformly convex.

1. Introduction. In 1950, R. C. James [J1] constructed a Banach space which is now called the James space. This space, along with its many variants (such as the James tree space [J2]) and their duals and preduals, have been a rich source for further research and results (both positive ones and counterexamples), answering many questions, several of which date back to Banach [B, 1932]. See [FG] for a splendid survey of such spaces.

This paper's main result, Theorem 5 , shows that the dual $J T^{*}$ of the James tree space $J T$ is asymptotically uniformly convex. (See Section 2 for definitions.)

Schachermayer [S, Theorem 4.1] showed that $J T^{*}$ has the Kadec-Klee property. It follows from Theorem 5 of this paper that $J T^{*}$ enjoys the uniform Kadec-Klee property. Of course, the same can be said about the (unique) predual $J T_{*}$ of $J T$. In fact, Theorem 3 shows that the modulus of asymptotic convexity of $J T_{*}$ is of power type 3 .

Johnson, Lindenstrauss, Preiss, and Schechtman [JLPS] showed that an asymptotically uniformly convex space has the point of continuity property and asked whether an asymptotically uniformly convex space has the Radon-Nikodým property. It is well known that both $J T_{*}$ and $J T^{*}$ have the point of continuity property yet fail the Radon-Nikodým property. It follows from Theorem 5 of this paper that $J T^{*}$ is an asymptotically uniformly convex (dual) space without the Radon-Nikodým property. Thus $J T_{*}$ is a separable asymptotically uniformly convex space without the Radon-Nikodým property. To the best of the author's knowledge, these are the first known examples of asymptotically uniformly convex spaces without the RadonNikodým property.

2000 Mathematics Subject Classification: 46B20, 46B22, 46 B99.

Key words and phrases: James tree space, asymptotically uniformly convex, RNP. 
2. Definitions and notation. Throughout this paper $\mathfrak{X}$ denotes an arbitrary (infinite-dimensional real) Banach space. If $\mathfrak{X}$ is a Banach space, then $\mathfrak{X}^{*}$ is its dual space, $B(\mathfrak{X})$ is its (closed) unit ball, $S(\mathfrak{X})$ is its unit sphere, $\widehat{\imath}: \mathfrak{X} \rightarrow \mathfrak{X}^{* *}$ is the natural point-evaluation isometric embedding, $\widehat{x}=\widehat{\imath}(x)$ and $\widehat{\mathfrak{X}}=\widehat{\imath}(\mathfrak{X})$. If $Y$ is a subset of $\mathfrak{X}$, then $[Y]$ is the closed linear span of $Y$ and

$$
\begin{aligned}
\mathfrak{N}(\mathfrak{X}) & =\left\{\left[x_{i}^{*}\right]_{1 \leq i \leq n}^{\top}: x_{i}^{*} \in \mathfrak{X}^{*} \text { and } n \in \mathbb{N}\right\}, \\
\mathcal{W}\left(\mathfrak{X}^{*}\right) & =\left\{\left[x_{i}\right]_{1 \leq i \leq n}^{\perp}: x_{i} \in \mathfrak{X} \text { and } n \in \mathbb{N}\right\} .
\end{aligned}
$$

Thus $\mathfrak{N}(\mathfrak{X})$ is the collection of (norm-closed) finite-codimensional subspaces of $\mathfrak{X}$ while $\mathcal{W}\left(\mathfrak{X}^{*}\right)$ is the collection of weak-star closed finite-codimensional subspaces of $\mathfrak{X}^{*}$. All notation and terminology, not otherwise explained, are as in [DU, LT1, LT2].

The modulus of convexity $\delta_{\mathfrak{X}}:[0,2] \rightarrow[0,1]$ of $\mathfrak{X}$ is

$$
\delta_{\mathfrak{X}}(\varepsilon)=\inf \left\{1-\left\|\frac{x+y}{2}\right\|: x, y \in S(\mathfrak{X}) \text { and }\|x-y\| \geq \varepsilon\right\}
$$

and $\mathfrak{X}$ is uniformly convex $(U C)$ if and only if $\delta_{\mathfrak{X}}(\varepsilon)>0$ for each $\varepsilon \in(0,2]$. The modulus of asymptotic convexity $\bar{\delta}_{\mathfrak{X}}:[0,1] \rightarrow[0,1]$ of $\mathfrak{X}$ is

$$
\bar{\delta}_{\mathfrak{X}}(\varepsilon)=\inf _{x \in S(\mathfrak{X})} \sup _{\mathcal{Y} \in \mathfrak{N}(\mathfrak{X})} \inf _{y \in S(\mathcal{Y})}[\|x+\varepsilon y\|-1]
$$

and $\mathfrak{X}$ is asymptotically uniformly convex $(A U C)$ if and only if $\bar{\delta}_{\mathfrak{X}}(\varepsilon)>0$ for each $\varepsilon$ in $(0,1]$.

A space $\mathfrak{X}$ has the Kadec-Klee $(K K)$ property provided the relative norm and weak topologies on $B(\mathfrak{X})$ coincide on $S(\mathfrak{X})$. A space $\mathfrak{X}$ has the uniform Kadec-Klee $(U K K)$ property provided for each $\varepsilon>0$ there exists $\delta>0$ such that every $\varepsilon$-separated weakly convergent sequence $\left\{x_{n}\right\}$ in $B(\mathfrak{X})$ converges to an element of norm less than $1-\delta$.

Related to the above geometric isometric properties are the following geometric isomorphic properties.

- $\mathfrak{X}$ has the Radon-Nikodým property $(R N P)$ provided each bounded subset of $\mathfrak{X}$ has nonempty slices of arbitrarily small diameter.

- $\mathfrak{X}$ has the point of continuity property $(P C P)$ provided each bounded subset of $\mathfrak{X}$ has nonempty relatively weakly open subsets of arbitrarily small diameter.

- $\mathfrak{X}$ has the complete continuity property $(C C P)$ provided each bounded subset of $\mathfrak{X}$ is Bocce dentable.

Implications between these various properties are summarized in the diagram below. 


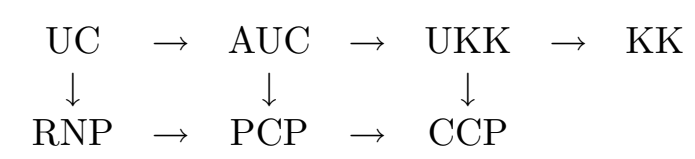

Helpful notation is

$$
\bar{\delta}_{\mathfrak{X}}(\varepsilon)=\inf _{x \in S(\mathfrak{X})} \bar{\delta}_{\mathfrak{X}}(\varepsilon, x)
$$

where

$$
\bar{\delta}_{\mathfrak{X}}(\varepsilon, x)=\sup _{\mathcal{Y} \in \mathfrak{N}(\mathfrak{X})} \inf _{y \in S(\mathcal{Y})}[\|x+\varepsilon y\|-1] .
$$

Note that, for each $x \in S(\mathfrak{X})$,

$$
\bar{\delta}_{\mathfrak{X}}(\varepsilon, x)=\sup _{\mathcal{Y} \in \mathfrak{N}(\mathfrak{X})} \inf _{\substack{y \in \mathcal{Y} \\\|y\| \geq \varepsilon}}[\|x+y\|-1]
$$

and so $\bar{\delta}_{\mathfrak{X}}(\varepsilon, x)$ is a nondecreasing function of $\varepsilon$. Thus $\bar{\delta}_{\mathfrak{X}}$ is a nondecreasing Lipschitz function with Lipschitz constant at most one. For any space $\mathfrak{X}$ and $\varepsilon \in[0,1]$,

$$
\bar{\delta}_{\mathfrak{X}}(\varepsilon) \leq \varepsilon=\bar{\delta}_{\ell_{1}}(\varepsilon) ;
$$

thus, $\ell_{1}$ is, in some sense, the most asymptotically uniformly convex space.

Uniform convexity, the KK property, and the UKK property have been extensively studied (for example, see [DGZ, LT2]). Asymptotic uniform convexity has been examined explicitly in [JLPS, M] and implicitly in [GKL, KOS]. The RNP, PCP, and CCP have also been extensively studied (for example, see [DU, GGMS, G1, G2]).

The JT space is constructed on a (binary) tree

$$
\mathcal{T}=\bigcup_{n=0}^{\infty} \Delta_{n}
$$

where $\Delta_{n}$ is the $n$th level of the tree; thus,

$$
\Delta_{0}=\{\emptyset\} \quad \text { and } \quad \Delta_{n}=\{-1,+1\}^{n}
$$

for each $n \in \mathbb{N}$. The finite tree $\mathcal{T}_{N}$ up through level $N \in \mathbb{N} \cup\{0\}$ is

$$
\mathcal{T}_{N}=\bigcup_{n=0}^{N} \Delta_{n} .
$$

The tree $\mathcal{T}$ is equipped with its natural (tree) ordering: if $t_{1}$ and $t_{2}$ are elements of $\mathcal{T}$, then $t_{1}<t_{2}$ provided one of the following holds:

1. $t_{1}=\emptyset$ and $t_{2} \neq \emptyset$,

2. for some $n, m \in \mathbb{N}$,

$$
t_{1}=\left(\varepsilon_{1}^{1}, \varepsilon_{2}^{1}, \ldots, \varepsilon_{n}^{1}\right) \text { and } t_{2}=\left(\varepsilon_{1}^{2}, \varepsilon_{2}^{2}, \ldots, \varepsilon_{m}^{2}\right)
$$

with $n<m$ and $\varepsilon_{i}^{1}=\varepsilon_{i}^{2}$ for each $1 \leq i \leq n$. 
A (finite) segment of $\mathcal{T}$ is a linearly ordered subset $\left\{t_{n}, t_{n+1}, \ldots, t_{n+k}\right\}$ of $\mathcal{T}$ where $t_{i} \in \Delta_{i}$ for each $n \leq i \leq n+k$. A branch of $\mathcal{T}$ is a linearly ordered subset $\left\{t_{0}, t_{1}, t_{2}, \ldots\right\}$ of $\mathcal{T}$ where $t_{i} \in \Delta_{i}$ for each $i \in \mathbb{N} \cup\{0\}$.

The James tree space $J T$ is the completion of the space of finitely supported functions $x: \mathcal{T} \rightarrow \mathbb{R}$ with respect to the norm

$$
\|x\|_{J T}=\sup \left\{\left[\sum_{i=1}^{n}\left|\sum_{t \in S_{i}} x_{t}\right|^{2}\right]^{1 / 2}: S_{1}, \ldots, S_{n} \text { are disjoint segments of } \mathcal{T}\right\} .
$$

By lexicographically ordering $\mathcal{T}$, the sequence $\left\{\eta_{t}\right\}_{t \in \mathcal{T}}$ in $J T$, where

$$
\eta_{t}(s)= \begin{cases}1 & \text { if } t=s \\ 0 & \text { if } t \neq s,\end{cases}
$$

forms a monotone boundedly complete (Schauder) basis of JT with biorthogonal functions $\left\{\eta_{t}^{*}\right\}_{t \in \mathcal{T}}$ in $J T^{*}$. Thus $\widehat{J T_{*}}=\left[\eta_{t}^{*}\right]_{t \in \mathcal{T}}$.

For $N, M \in \mathbb{N} \cup\{0\}$ with $N \leq M$, the restriction maps from $J T$ to $J T$ given by

$$
\begin{aligned}
\pi_{N}(x) & =\sum_{t \in \Delta_{N}} \eta_{t}^{*}(x) \eta_{t}, \\
\pi_{[N, M]}(x) & =\sum_{t \in \cup_{i=N}^{M} \Delta_{i}} \eta_{t}^{*}(x) \eta_{t}, \\
\pi_{[N, \omega)}(x) & =\sum_{t \in \cup_{i=N}^{\infty} \Delta_{i}} \eta_{t}^{*}(x) \eta_{t}
\end{aligned}
$$

are each contractive projections (by the nature of the norm on $J T$ ); thus, so are their adjoints.

Let $\Gamma$ be the set of all branches of $\mathcal{T}$. Then [LS, Theorem 1] the mapping $\pi_{\infty}: J T^{*} \rightarrow \ell_{2}(\Gamma)$ given by

$$
\pi_{\infty}\left(x^{*}\right)=\left\{\lim _{t \in B} x^{*}\left(\eta_{t}\right)\right\}_{B \in \Gamma}
$$

is an isometric quotient mapping with kernel $\widehat{J T_{*}}$. Also, for each $x^{*} \in J T^{*}$,

$$
\begin{aligned}
\left\|x^{*}\right\| & =\lim _{N \rightarrow \infty}\left\|\pi_{[0, N]}^{*} x^{*}\right\|, \\
\left\|\pi_{\infty} x^{*}\right\| & =\lim _{N \rightarrow \infty}\left\|\pi_{[N, \omega)}^{*} x^{*}\right\|=\lim _{N \rightarrow \infty}\left\|\pi_{N}^{*} x^{*}\right\|,
\end{aligned}
$$

by the weak-star lower semicontinuity of the norm on $J T^{*}$.

To show that $J T^{*}$ has the Kadec-Klee property, Schachermayer calculated the two quantitative bounds below.

FACT 1 [S, Lemma 3.8]. Let $f_{1}:(0,1) \rightarrow(0, \infty)$ be a continuous strictly increasing function satisfying $f_{1}(t)<2^{-10} t^{3}$ for each $t \in(0,1)$. Let $N \in \mathbb{N}$ and $z^{*} \in J T^{*}$. If

$$
\left[1-f_{1}(t)\right]\left\|z^{*}\right\|<\left\|\pi_{[0, N]}^{*} z^{*}\right\|
$$


then

$$
\left\|\pi_{[N, \omega)}^{*} z^{*}\right\|<\left\|\pi_{N}^{*} z^{*}\right\|+t\left\|z^{*}\right\| .
$$

FACT 2 [S, Lemma 3.11]. Let $f_{2}:(0,1) \rightarrow(0, \infty)$ be a continuous strictly increasing function satisfying $f_{2}(t)<2^{-26} t^{5}$ for each $t \in(0,1)$. Let $N \in \mathbb{N}$ and $\varepsilon_{0} \in(0,1)$ and $\widetilde{x}^{*}, \widetilde{u}^{*} \in J T^{*}$. If

$$
\begin{aligned}
& \left\|\pi_{[N, \omega)}^{*} \widetilde{x}^{*}\right\| \leq 1, \\
& \left\|\pi_{N}^{*} \widetilde{x}^{*}\right\|>1-f_{2}\left(\varepsilon_{0}\right), \\
& \left\|\pi_{\infty} \widetilde{x}^{*}\right\|>1-f_{2}\left(\varepsilon_{0}\right), \\
& \left\|\pi_{[N, \omega)}^{*}\left(\widetilde{x}^{*}+\widetilde{u}^{*}\right)\right\| \leq 1, \\
& \left\|\pi_{N}^{*} \widetilde{u}^{*}\right\|<f_{2}\left(\varepsilon_{0}\right), \\
& \left\|\pi_{\infty} \widetilde{u}^{*}\right\|<f_{2}\left(\varepsilon_{0}\right),
\end{aligned}
$$

then

$$
\left\|\pi_{[N, \omega)}^{*} \widetilde{u}^{*}\right\|<\varepsilon_{0}
$$

3. Results. Theorem 3 shows that the modulus of asymptotic convexity of $J T_{*}$ is of power type 3 . Its proof uses Fact 1 .

THEOREM 3. There exists a positive constant $k$ so that

$$
\bar{\delta}_{J T_{*}}(\varepsilon) \geq k \varepsilon^{3}
$$

for each $\varepsilon \in(0,1]$. Thus $J T_{*}$ is asymptotically uniformly convex.

Proof. Fix $c \in\left(0,2^{-10}\right)$ and find $k$ so that

$$
0<k(1+k)^{2} \leq c .
$$

Fix $\varepsilon \in(0,1)$ and a finitely supported $x_{*} \in S\left(J T_{*}\right)$. It suffices to show that

$$
\bar{\delta}_{J T_{*}}\left(\varepsilon, x_{*}\right) \geq k \varepsilon^{3} \text {. }
$$

Find $N \in \mathbb{N}$ so that $\pi_{[0, N-1]}^{*} \widehat{x}_{*}=\widehat{x}_{*}$ and let $\mathcal{Y}=\left[\eta_{t}\right]_{t \in \mathcal{T}_{N}}^{\top}$. Fix $y_{*} \in S(\mathcal{Y})$. Assume that

$$
\left\|x_{*}+\varepsilon y_{*}\right\|-1<k \varepsilon^{3} .
$$

Then

$$
\left[1-\frac{k \varepsilon^{3}}{1+k \varepsilon^{3}}\right]\left\|\widehat{x}_{*}+\varepsilon \widehat{y}_{*}\right\|<1=\left\|\pi_{[0, N]}^{*}\left(\widehat{x}_{*}+\varepsilon \widehat{y}_{*}\right)\right\| .
$$

Thus by Fact 1 , with $f_{1}(t)=c t^{3}$,

$$
\left\|\pi_{[N, \omega)}^{*}\left(\widehat{x}_{*}+\varepsilon \widehat{y}_{*}\right)\right\|<\left\|\pi_{N}^{*}\left(\widehat{x}_{*}+\varepsilon \widehat{y}_{*}\right)\right\|+f_{1}^{-1}\left(\frac{k \varepsilon^{3}}{1+k \varepsilon^{3}}\right)\left\|\left(\widehat{x}_{*}+\varepsilon \widehat{y}_{*}\right)\right\|
$$

and so

$$
\varepsilon<\left[1+k \varepsilon^{3}\right] f_{1}^{-1}\left(\frac{k \varepsilon^{3}}{1+k \varepsilon^{3}}\right)
$$


But inequality (3) is equivalent to

$$
c^{1 / 3}<k^{1 / 3}\left(1+k \varepsilon^{3}\right)^{2 / 3},
$$

which contradicts (1). Thus $\left\|x_{*}+\varepsilon y_{*}\right\|-1 \geq k \varepsilon^{3}$ and so (2) holds.

A modification of the proof of Theorem 3 shows that, for each $\varepsilon \in(0,1)$, the $\bar{\delta}_{J T^{*}}\left(\varepsilon, x^{*}\right)$ stays uniformly bounded below from zero for $x^{*} \in S\left(J T^{*}\right)$ whose $\left\|\pi_{\infty} x^{*}\right\|$ is small. Recall that if $x_{*} \in J T_{*}$ then $\left\|\pi_{\infty} \widehat{x}_{*}\right\|=0$.

Lemma 4. For each $\varepsilon \in(0,1)$ there exists $\eta=\eta(\varepsilon)>0$ so that

$$
\inf _{\substack{x^{*} \in S\left(J T^{*}\right) \\\left\|\pi_{\infty} x^{*}\right\| \leq \eta}} \sup _{\mathcal{Y} \in \mathcal{W}\left(J T^{*}\right)} \inf _{y^{*} \in S(\mathcal{Y})}\left[\left\|x^{*}+\varepsilon y^{*}\right\|-1\right]>0 .
$$

Proof. Fix $\varepsilon \in(0,1)$. With the notation of Fact 1 , find $\delta, \eta_{2}>0$ so that

$$
4 \eta_{2}+\frac{\delta}{1-f_{1}(\delta)}<\varepsilon
$$

Fix $x^{*} \in S\left(J T^{*}\right)$ with $\left\|\pi_{\infty} x^{*}\right\| \equiv b \leq \eta_{2}$. It suffices to show that

$$
\sup _{\mathcal{Y} \in \mathcal{W}\left(J T^{*}\right)} \inf _{y^{*} \in S(\mathcal{Y})}\left\|x^{*}+\varepsilon y^{*}\right\| \geq \frac{1}{1-f_{1}(\delta)} .
$$

Fix $\eta_{1} \in(0,1)$. Find $N \in \mathbb{N}$ so that

$$
1-\eta_{1} \leq\left\|\pi_{[0, N]}^{*} x^{*}\right\| \quad \text { and } \quad\left\|\pi_{[N, \omega)}^{*} x^{*}\right\|<b+\eta_{2}
$$

and let $\mathcal{Y}=\left[\eta_{t}\right]_{t \in \mathcal{T}_{N}}^{\perp}$. Fix $y^{*} \in S(\mathcal{Y})$.

Assume that

$$
\left\|x^{*}+\varepsilon y^{*}\right\|<\frac{1-\eta_{1}}{1-f_{1}(\delta)} .
$$

Then

$$
\left[1-f_{1}(\delta)\right]\left\|x^{*}+\varepsilon y^{*}\right\|<\left\|\pi_{[0, N]}^{*} x^{*}\right\|=\left\|\pi_{[0, N]}^{*}\left(x^{*}+\varepsilon y^{*}\right)\right\| .
$$

Thus by Fact 1 ,

$$
\left\|\pi_{[N, \omega)}^{*}\left(x^{*}+\varepsilon y^{*}\right)\right\|<\left\|\pi_{N}^{*}\left(x^{*}+\varepsilon y^{*}\right)\right\|+\delta\left\|\left(x^{*}+\varepsilon y^{*}\right)\right\|
$$

and so

$$
\varepsilon-\left(b+\eta_{2}\right)<\left(b+\eta_{2}\right)+\frac{\delta}{1-f_{1}(\delta)} .
$$

But $b \leq \eta_{2}$ and so

$$
\varepsilon<4 \eta_{2}+\frac{\delta}{1-f_{1}(\delta)}
$$

A contradiction, thus

$$
\left\|x^{*}+\varepsilon y^{*}\right\| \geq \frac{1-\eta_{1}}{1-f_{1}(\delta)} .
$$

Since $\eta_{1}>0$ was arbitrary, inequality (4) holds. 
Thus to show that $J T^{*}$ is asymptotically uniformly convex, one just needs to examine $\bar{\delta}_{J T^{*}}\left(\varepsilon, x^{*}\right)$ for $x^{*} \in S\left(J T^{*}\right)$ whose $\left\|\pi_{\infty} x^{*}\right\|$ is not small. Fact 2 is used for this case.

THEOREM 5. JT* is asymptotically uniformly convex.

Proof. Fix $\varepsilon \in(0,1)$ and let $\varepsilon_{0}=\varepsilon / 4$. Let $f_{1}:(0,1) \rightarrow\left(0,2^{-12}\right)$ be given by $f_{1}(t)=2^{-12} t^{3}$ and $f_{2}$ be a function satisfying the hypothesis in Fact 2 . Find $\delta, \eta_{2}>0$ so that

$$
4 \eta_{2}+\frac{\delta}{1-f_{1}(\delta)}<\varepsilon
$$

Next find $\gamma_{i}>0$ and $\tau>1$ so that

$$
\begin{aligned}
& \gamma_{3}<\gamma_{2}<1 / 2 \\
& \tau \leq \frac{\left(1-\gamma_{1}\right)\left(1-\gamma_{2}\right)}{1-f_{2}\left(\varepsilon_{0}\right)}, \\
& \tau<\frac{1-\gamma_{2}}{\sqrt{1-f_{2}^{2}\left(\varepsilon_{0}\right)}} \\
& \tau \leq \frac{\eta_{2}^{3} \gamma_{3}^{3}}{2^{15}\left(1-\gamma_{2}\right)^{3}}-\gamma_{4}+1, \\
& \frac{\tau-1+\gamma_{4}}{\tau}<f_{1}(1) \\
& \tau \leq \frac{1}{1-f_{1}(\delta)}
\end{aligned}
$$

Fix $x^{*} \in S\left(J T^{*}\right)$. It suffices to show that

$$
\sup _{\mathcal{Y} \in \mathfrak{N}\left(J T^{*}\right)} \inf _{y \in S(\mathcal{Y})}\left\|x^{*}+\varepsilon y^{*}\right\| \geq \tau .
$$

Let $\left\|\pi_{\infty} x^{*}\right\| \equiv b$. If $b \leq \eta_{2}$, then by the proof of Lemma 4 and (10), inequality (11) holds. So let $b>\eta_{2}$. Find $N \in \mathbb{N}$ so that

$$
\begin{aligned}
&\left(1-\gamma_{1}\right) b<\left\|\pi_{N}^{*} x^{*}\right\| \leq\left\|\pi_{[N, \omega)}^{*} x^{*}\right\|<b\left(\frac{1-\gamma_{3}}{1-\gamma_{2}}\right)<\frac{b}{1-\gamma_{2}}, \\
& 1-\gamma_{4}<\left\|\pi_{[0, N]}^{*} x^{*}\right\| .
\end{aligned}
$$

Let $g_{x^{*}} \in J T^{* *}$ be the functional given by

$$
g_{x^{*}}\left(z^{*}\right)=\left\langle\pi_{\infty} z^{*}, \pi_{\infty} x^{*}\right\rangle_{H_{2}}
$$

where the inner product is the natural inner product on $\ell_{2}(\Gamma)$. Let

$$
\mathcal{Y}=\left[\eta_{t}\right]_{t \in \mathcal{T}_{N}}^{\perp} \cap\left[g_{x^{*}}\right]^{\top}
$$

and fix $y^{*} \in S(\mathcal{Y})$.

Assume that

$$
\left\|x^{*}+\varepsilon y^{*}\right\|<\tau
$$


It suffices to find a contradiction to (14). Towards this, let

$$
\widetilde{x}^{*}=\frac{1-\gamma_{2}}{\tau b} x^{*} \text { and } \quad \widetilde{y}^{*}=\frac{1-\gamma_{2}}{\tau b} y^{*} .
$$

It suffices to show (keeping the same notation but with $\widetilde{u}^{*}=\varepsilon \widetilde{y}^{*}$ ) that conditions (2.1) through (2.6) of Fact 2 hold; for then condition (2.7) holds and so by (5),

$$
\varepsilon_{0}>\left\|\pi_{[N, \omega)}^{*} \varepsilon \widetilde{y}^{*}\right\|=\frac{1-\gamma_{2}}{\tau b} \varepsilon \geq \frac{\varepsilon}{4}=\varepsilon_{0} .
$$

Condition (2.1) follows from (12) since

$$
\left\|\pi_{[N, \omega)}^{*} \widetilde{x}^{*}\right\| \leq \frac{1-\gamma_{2}}{\tau b} \cdot \frac{b}{1-\gamma_{2}} \leq 1 .
$$

Condition (2.2) follows from (12) and (6) since

$$
\left\|\pi_{N}^{*} \widetilde{x}^{*}\right\|>\frac{1-\gamma_{2}}{\tau b}\left(1-\gamma_{1}\right) b=\frac{\left(1-\gamma_{1}\right)\left(1-\gamma_{2}\right)}{\tau} \geq 1-f_{2}\left(\varepsilon_{0}\right) .
$$

Towards condition (2.3), note that by (7),

$$
\left\|\pi_{\infty} \widetilde{x}^{*}\right\|=\frac{1-\gamma_{2}}{\tau b} b=\frac{1-\gamma_{2}}{\tau}>\sqrt{1-f_{2}^{2}\left(\varepsilon_{0}\right)}
$$

and so

$$
\left\|\pi_{\infty} \widetilde{x}^{*}\right\|>1-f_{2}\left(\varepsilon_{0}\right) .
$$

Towards condition (2.4), note that by (14) and (13),

$$
\left\|x^{*}+\varepsilon y^{*}\right\|<\frac{\tau}{1-\gamma_{4}}\left\|\pi_{[0, N]}^{*}\left(x^{*}+\varepsilon y^{*}\right)\right\| .
$$

Thus by Fact 1 and (9),

$$
\begin{aligned}
\left\|\pi_{[N, \omega)}^{*}\left(x^{*}+\varepsilon y^{*}\right)\right\|< & \left\|\pi_{N}^{*}\left(x^{*}+\varepsilon y^{*}\right)\right\| \\
& +f_{1}^{-1}\left(\frac{\tau-1+\gamma_{4}}{\tau}\right)\left\|\left(x^{*}+\varepsilon y^{*}\right)\right\| \\
\leq & b \frac{1-\gamma_{3}}{1-\gamma_{2}}+\tau 2^{4}\left(\frac{\tau-1+\gamma_{4}}{\tau}\right)^{1 / 3} .
\end{aligned}
$$

Thus condition (2.4) holds provided

$$
b \frac{1-\gamma_{3}}{1-\gamma_{2}}+\tau 2^{4}\left(\frac{\tau-1+\gamma_{4}}{\tau}\right)^{1 / 3} \leq \frac{\tau b}{1-\gamma_{2}},
$$

or equivalently

$$
\tau^{2 / 3}\left(\tau-1+\gamma_{4}\right)^{1 / 3} \leq \frac{b\left(\tau-1+\gamma_{3}\right)}{2^{4}\left(1-\gamma_{2}\right)}
$$


But by (8) and since $b>\eta_{2}$,

$$
\begin{aligned}
\tau^{2 / 3}\left(\tau-1+\gamma_{4}\right)^{1 / 3} & \leq 2\left(\tau-1+\gamma_{4}\right)^{1 / 3} \leq \frac{2 \eta_{2} \gamma_{3}}{2^{5}\left(1-\gamma_{2}\right)} \\
& \leq \frac{b \gamma_{3}}{2^{4}\left(1-\gamma_{2}\right)} \leq \frac{b\left(\tau-1+\gamma_{3}\right)}{2^{4}\left(1-\gamma_{2}\right)}
\end{aligned}
$$

Thus condition (2.4) holds.

Condition (2.5) follows from the fact that $y^{*} \in\left[\eta_{t}\right]_{t \in \mathcal{T}_{N}}^{\perp}$. Towards condition (2.6), since $y^{*} \in\left[g_{x^{*}}\right]^{\top}$, the vectors $\pi_{\infty} \widetilde{y}^{*}$ and $\pi_{\infty} \widetilde{x}^{*}$ are orthogonal in $\ell_{2}(\Gamma)$ and so

$$
\left\|\pi_{\infty} \varepsilon \widetilde{y}^{*}\right\|^{2}=\left\|\pi_{\infty}\left(\widetilde{x}^{*}+\varepsilon \widetilde{y}^{*}\right)\right\|^{2}-\left\|\pi_{\infty} \widetilde{x}^{*}\right\|^{2} ;
$$

but $\pi_{\infty}=\pi_{\infty} \pi_{[N, \omega)}^{*}$ and so by condition (2.4) and (15),

$$
\begin{aligned}
\left\|\pi_{\infty} \varepsilon \widetilde{y}^{*}\right\|^{2} & \leq\left\|\pi_{[N, \omega)}^{*}\left(\widetilde{x}^{*}+\varepsilon \widetilde{y}^{*}\right)\right\|^{2}-\left\|\pi_{\infty} \widetilde{x}^{*}\right\|^{2} \\
& <1-\left[1-f_{2}^{2}\left(\varepsilon_{0}\right)\right]=f_{2}^{2}\left(\varepsilon_{0}\right) .
\end{aligned}
$$

Thus condition (2.6) holds.

The proof in [JLPS] that an asymptotically uniformly convex space has the PCP shows that if $\bar{\delta}_{\mathfrak{X}}(\varepsilon)>0$ for each $\varepsilon \in(0,1]$ then $\mathfrak{X}$ has the PCP. A bit more can be said.

Proposition 6. If $\bar{\delta}_{\mathfrak{X}}(1 / 2)>0$ then $\mathfrak{X}$ has the PCP.

The proof of Proposition 6 uses the following (essentially known) lemma.

Lemma 7. Let $\mathfrak{X}$ be a space without the PCP and $0<\varepsilon<1$. Then there is a closed subset $A$ of $\mathfrak{X}$ so that

(1) each (nonempty) relatively weakly open subset of $A$ has diameter larger than $1-\varepsilon$,

(2) $\sup \{\|a\|: a \in A\}=1$.

Proof. Let $\mathfrak{X}$ fail the PCP and $0<\varepsilon<1$. By a standard argument (e.g., see [SSW, Prop. 4.10]), there is a closed subset $\widetilde{A}$ of $\mathfrak{X}$ of diameter one such that each (nonempty) relatively weakly open subset of $\widetilde{A}$ has diameter larger than $1-\varepsilon$. Without loss of generality $0 \in \widetilde{A}$ (just consider a translate of $\widetilde{A}$ ). Let

$$
b=\sup \{\|x\|: x \in \widetilde{A}\} \quad \text { and } \quad A=\widetilde{A} / b .
$$

Note that $0<b \leq 1$. If $V$ is a (nonempty) relatively weakly open subset of $A$, then $b V$ is a relatively weakly open subset of $\widetilde{A}$ and so

Thus $A$ does the job.

$$
\operatorname{diam} V=\frac{1}{b} \operatorname{diam} b V>1-\varepsilon .
$$


Proof of Proposition 6. Let $\mathfrak{X}$ be a Banach space without the PCP. Fix $t \in(0,1 / 2)$ and $\delta \in(0, t)$. It suffices to show that $\bar{\delta}_{\mathfrak{X}}(t) \leq 2 \delta$.

Find a subset $A$ of $\mathfrak{X}$ which satisfies the conditions of Lemma 7 with $\varepsilon=1-2 t$ and find $a \in A$ so that

$$
\left\|\frac{a}{\|a\|}-a\right\|<\delta
$$

Let $\mathcal{Y} \in \mathfrak{N}(\mathfrak{X})$. It suffices to show that

$$
\inf _{\substack{y \in \mathcal{Y} \\\|y\| \geq t}}\left[\left\|\frac{a}{\|a\|}+y\right\|-1\right] \leq 2 \delta .
$$

By condition (1) of Lemma 7 there exists $x \in A$ so that $\|x-a\| \geq t$ and $x-a$ is almost in $\mathcal{Y}$; thus, by a standard perturbation argument (e.g., see [GJ, Lemma 2]) there exists $y \in \mathcal{Y}$ so that

$$
\|y\| \geq t \quad \text { and } \quad\|y-(x-a)\|<\delta .
$$

Thus

$$
\left\|\frac{a}{\|a\|}+y\right\| \leq\left\|\frac{a}{\|a\|}-a\right\|+\|y-x+a\|+\|x\|<1+2 \delta .
$$

Thus $\bar{\delta}_{\mathfrak{X}}(1 / 2)=0$.

The observation below formalizes an essentially known fact, which to the best of the author's knowledge, has not appeared in print as such. Recall that the modulus of asymptotic smoothness $\bar{\varrho}_{\mathfrak{X}}:[0,1] \rightarrow[0,1]$ of $\mathfrak{X}$ is

$$
\bar{\varrho}_{\mathfrak{X}}(\varepsilon)=\sup _{x \in S(\mathfrak{X})} \inf _{\mathcal{Y} \in \mathfrak{N}(\mathfrak{X})} \sup _{y \in S(\mathcal{Y})}[\|x+\varepsilon y\|-1]
$$

and $\mathfrak{X}$ is asymptotically uniformly smooth if and only if $\lim _{\varepsilon \rightarrow 0^{+}} \varrho_{\mathfrak{X}}(\varepsilon) / \varepsilon=0$. Also, $L_{p}(\mathfrak{X})$ is the Lebesgue-Bochner space of strongly measurable $\mathfrak{X}$-valued functions defined on a separable nonatomic probability space, equipped with its usual norm.

Observation 8. Let $1<p<\infty$. For a Banach space $\mathfrak{X}$, the following are equivalent.

(1) $\mathfrak{X}$ is uniformly convexifiable.

(2) $L_{p}(\mathfrak{X})$ is uniformly convexifiable.

(3) $L_{p}(\mathfrak{X})$ is asymptotically uniformly convexifiable.

(4) $L_{p}(\mathfrak{X})$ admits an equivalent UKK norm.

(5) $L_{p}(\mathfrak{X})$ is asymptotically uniformly smoothable.

Proof. Let $1<p<\infty$ and $\mathfrak{X}$ be a Banach space.

That (1) through (4) are equivalent and that (2) implies (5) follows easily from the following known facts about a Banach space $\mathcal{Y}$ :

(i) $\mathcal{Y}$ is uniformly convex if and only if $L_{p}(\mathcal{Y})$ is $[\mathrm{Mc}]$. 
(ii) $\mathcal{Y}$ is uniformly convexifiable if and only if $L_{p}(\mathcal{Y})$ admits an equivalent UKK norm [DGK, Theorem 4].

(iii) $\mathcal{Y}$ is uniformly convexifiable if and only if $\mathcal{Y}$ is uniformly smoothable (cf. [DU, page 144]).

Towards showing that $(5)$ implies $(1)$, let $L_{p}(\mathfrak{X})$ be asymptotically uniformly smoothable and $\mathfrak{X}_{0}$ be a separable subspace of $\mathfrak{X}$. It suffices to show that $\mathfrak{X}_{0}$ is uniformly convexifiable (cf. [DGZ, Remark IV.4.4]).

It follows from [GKL, Proposition 2.6] that if $\mathcal{Y}$ is separable, then $\mathcal{Y}$ is asymptotically uniformly smooth if and only if $\mathcal{Y}^{*}$ has the $\mathrm{UKK}^{*}$ property. Thus $\left[L_{p}\left(\mathfrak{X}_{0}\right)\right]^{*}$ admits an equivalent $\mathrm{UKK}^{*}$ norm. But $\ell_{1}$ cannot embed into $L_{p}\left(\mathfrak{X}_{0}\right)$ since $L_{p}\left(\mathfrak{X}_{0}\right)$ is asymptotically uniformly smoothable and so $\left[L_{p}\left(\mathfrak{X}_{0}\right)\right]^{*}$ is asymptotically weak* uniformly convexifiable and so is also asymptotically uniformly convexifiable. Thus $L_{q}\left(\mathfrak{X}_{0}^{*}\right)$ is asymptotically uniformly convexifiable where $1 / p+1 / q=1$. From $(3) \Rightarrow(1)$ it follows that $\mathfrak{X}_{0}^{*}$ is uniformly convexifiable and hence so is $\mathfrak{X}_{0}$.

Acknowledgements. The author thanks William B. Johnson and Thomas Schlumprecht for fruitful discussions on asymptotic uniform convexity at the NSF Workshop in Linear Analysis and Probability, Texas A\&M University, during the Summer of 1999.

\section{References}

[B] S. Banach, Théorie des opérations linéaires, Éditions Jacques Gabay, Sceaux, 1993, reprint of the 1932 original.

[DGZ] R. Deville, G. Godefroy and V. Zizler, Smoothness and Renormings in Banach Spaces, Longman Sci. \& Tech., Harlow, 1993.

[DU] J. Diestel and J. J. Uhl, Jr., Vector Measures, Math. Surveys 15, Amer. Math. Soc., Providence, RI, 1977.

[DGK] S. J. Dilworth, Maria Girardi and D. Kutzarova, Banach spaces which admit a norm with the uniform Kadec-Klee property, Studia Math. 112 (1995), 267-277.

[FG] H. Fetter and B. Gamboa de Buen, The James Forest, Cambridge Univ. Press, Cambridge, 1997.

[GGMS] N. Ghoussoub, G. Godefroy, B. Maurey and W. Schachermayer, Some topological and geometrical structures in Banach spaces, Mem. Amer. Math. Soc. 378 (1987).

[G1] Maria Girardi, Dunford-Pettis operators on $L_{1}$ and the complete continuity property, Ph.D. dissertation, Univ. of Illinois at Urbana-Champaign, UrbanaChampaign, IL, 1990 (http://www.math.sc.edu/ girardi/).

[G2] - Dentability, trees, and Dunford-Pettis operators on $L_{1}$, Pacific J. Math. 148 (1991), 59-79.

[GJ] Maria Girardi and W. B. Johnson, Universal non-completely-continuous operators, Israel J. Math. 99 (1997), 207-219.

[GKL] G. Godefroy, N. J. Kalton and G. Lancien, Szlenk indices and uniform homeomorphisms, preprint (http://front.math.ucdavis.edu/math.FA/9911017/). 
[J1] R. C. James, Bases and reflexivity of Banach spaces, Ann. of Math. (2) 52 (1950), 518-527.

[J2] - A separable somewhat reflexive Banach space with nonseparable dual, Bull. Amer. Math. Soc. 80 (1974), 738-743.

[JLPS] W. B. Johnson, J. Lindenstrauss, D. Preiss and G. Schechtman, Almost Fréchet differentiability of Lipschitz mappings between infinite dimensional Banach spaces, preprint.

[KOS] H. Knaust, E. Odell and T. Schlumprecht, On asymptotic structure, the Szlenk index and UKK properties in Banach spaces, Positivity 3 (1999), 173-199.

[LS] J. Lindenstrauss and C. Stegall, Examples of separable spaces which do not contain $\ell_{1}$ and whose duals are non-separable, Studia Math. 54 (1975), 81-105.

[LT1] J. Lindenstrauss and L. Tzafriri, Classical Banach Spaces. I, Sequence Spaces, Ergeb. Math. Grenzgeb. 92, Springer, Berlin, 1977.

[LT2] -, -, Classical Banach Spaces. II, Function Spaces, Springer, Berlin, 1979.

[Mc] E. J. McShane, Linear functionals on certain Banach spaces, Proc. Amer. Math. Soc. 1 (1950), 402-408.

[M] V. D. Milman, Geometric theory of Banach spaces. II. Geometry of the unit ball, Uspekhi Mat. Nauk 26 (1971), no. 6, 73-149.

[S] W. Schachermayer, Some more remarkable properties of the James-tree space, in: Banach Space Theory (Iowa City, IA, 1987), Amer. Math. Soc., Providence, RI, 1989, 465-496.

[SSW] W. Schachermayer, A. Sersouri and E. Werner, Moduli of nondentability and the Radon-Nikodým property in Banach spaces, Israel J. Math. 65 (1989), 225-257.

Department of Mathematics

University of South Carolina

Columbia, SC 29208, U.S.A.

E-mail: girardi@math.sc.edu

Received May 4, 2000

Revised version March 15, 2001 